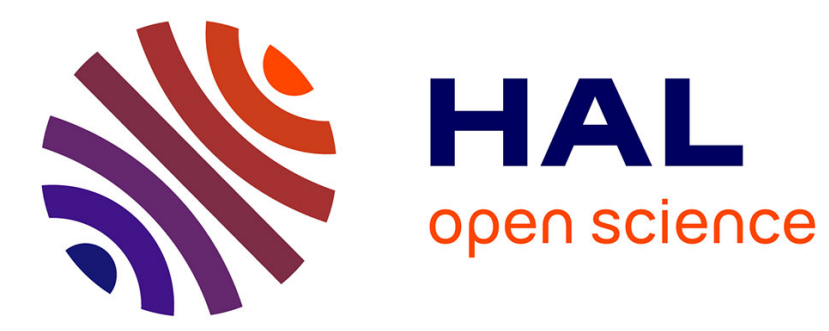

\title{
Human gait classification using MicroDoppler time-frequency signal representations
}

Bastien Lyonnet, Cornel Ioana, Moeness Null Amin

\section{To cite this version:}

Bastien Lyonnet, Cornel Ioana, Moeness Null Amin. Human gait classification using MicroDoppler time-frequency signal representations. Radar 2010 - IEEE International Radar Conference, May 2010, Arlington, Virginie, United States. Paper ID 9294. hal-00537392

\section{HAL Id: hal-00537392 https://hal.science/hal-00537392}

Submitted on 18 Nov 2010

HAL is a multi-disciplinary open access archive for the deposit and dissemination of scientific research documents, whether they are published or not. The documents may come from teaching and research institutions in France or abroad, or from public or private research centers.
L'archive ouverte pluridisciplinaire HAL, est destinée au dépôt et à la diffusion de documents scientifiques de niveau recherche, publiés ou non, émanant des établissements d'enseignement et de recherche français ou étrangers, des laboratoires publics ou privés. 


\section{Human gait classification using MicroDoppler time- frequency signal representations}

\author{
Bastien Lyonnet, Cornel Ioana \\ Grenoble Institute of Technology \\ GIPSA-lab, Signal-Image Department \\ Saint Martin d'Heres, France \\ [bastien.lyonnet,cornel.ioana]@gipsa-lab.grenoble-inp.fr
}

\author{
Moeness G. Amin \\ Villanova University \\ Center for Advanced Communications \\ Villanova, PA, United States of America \\ moeness.amin@villanova.edu
}

\begin{abstract}
A time-frequency classifier is applied for human gait classification. Time-frequency (t-f) quadratic distributions are used for time-frequency signal representations. Three specific motions are considered, corresponding to three different scenarios of arm motions which describe free and confined arm swings. The microDoppler signature in the time-frequency domain of each motion style is viewed as a feature and is incorporated in a distance-based classifications measured between the test data $t-f$ distribution and the training average $t-f$ distributions. It is shown that the time-frequency classifier performs properly, yielding low probability of classification errors, and its performance is rather insensitive to the type of time-frequency distribution employed. Among the possible distance measures used, which include the Correlation, Bhattacharyya and Kolmogorov, the Euclidean distance provided the best results.
\end{abstract}

\section{INTRODUCTION}

Human gait has complex articulation and reflects radar signals with Doppler modulations that reveal information about motion dynamics. The overall Doppler signature of a moving human, including breathing and heartbeat, which constitutes the biometric radars, has received significant interest during the last few years [1-5]. However, literature on human gait recognition using radar has often been concerned with the processing and extraction of MicroDoppler $(\mu \mathrm{D})$ rather than gait recognition or classification. Recognizing that time-frequency representations (TFRs) are an efficient tool for nonstationary signal classification, human gait analysis has typically been performed with joint time-frequency signal techniques [6]. In [12], scatter plots of stride and appendage/torso ratio vs. velocity are used as gait signature and a linear classifier is built to identify gender and human presence.

This paper considers the classification of the nature of the walking motion of humans using MicroDoppler radar returns represented by their time-frequency signatures. The time-frequency classifier used in this paper follows the work in [13] in which no specific feature is extracted, but rather the entire time-frequency representation is used. Unlike PCA and support vector machine based classifications [14,15], the entire time-frequency domain is employed in providing the distance measure.

This paper shows that the aspect angle plays a role in human gait classification. Specifically, in the case when the motion trajectory puts the human torso in the line of sight between the radar and one of the two arms. In this case, the classifier tends to confuse a one-arm obstructed motion as noarm motion.

\section{HUMAN GAIT REPRESENTATION}

Doppler spectrum is a complex response of gait cycle phase to incident radar illumination. We consider a point target situated at a distance $R(t)$. The target is moving towards the radar at a velocity $v(t)$. Let $s(t)$ represents the transmitted signal,

$$
s(t)=A \cos (2 \pi f t)
$$

The received signal is given by $r(t)$,

$$
\mathrm{r}(\mathrm{t})=\mathrm{A}^{\prime} \cos \left(2 \pi f\left(\mathrm{t}-2 \frac{\mathrm{R}(\mathrm{t})}{c}\right)\right)
$$

with $c$ the speed of light and $A^{\prime}$ a reflexion coefficient. If reflected from a moving target (limb) with an angle $\theta$ with the radar direction, then the reflected signal can be written as,

$$
r(t)=A^{\prime} \cos \left(2 \pi f\left(1+\frac{2 v(t)}{c}\right) t-\frac{4 \pi R(0)}{c}\right)
$$

The corresponding Doppler shift is

$$
f_{d}=f \frac{2 v(t)}{c}
$$


When scattering from the total human body is considered, the radar return becomes a superposition of the different moving parts : torso, arms, legs and feet. Each part has a different velocity and acceleration. If we consider $J$ different moving parts of the body, the received signal is,

$$
r(t)=\sum_{j=0}^{N} A_{j}^{\prime} \cos \left(2 \pi f\left(1+\frac{2 v_{j}(t)}{c}\right) t-\frac{4 \pi R_{j}(0)}{c}\right)
$$

Accordingly, the Doppler shift is a complex interaction of multiple Doppler shifts generated by the limb and torso movements.

It is noted that the maximum speed of a person walking is typically $1.5 \mathrm{~m} / \mathrm{sec}$ which is equivalent to $25 \mathrm{~Hz}$ frequency shift when using a Doppler radar at $2.5 \mathrm{GHz}$. Clearly, the ability to detect human gait depends on the Doppler signature and the power remaining after the MTI filtering for clutter removal is applied. The microDoppler generated by the movements of arms and legs is a highly nonstationary signal which can be revealed in the time-frequency domain using conventional or modern time-frequency signal representation techniques.

\section{THE ClASSIFICATION MODEL}

Define $d(.,$.$) as dissimilarity or distance measure and$ $C_{x}^{\varphi}(t, f)$ as time-frequency distribution from Cohen's class, characterized by its respective kernel function. The signal $x$ belong to one of the $N$ class of motions. In essence, as a classification rule, we assign $x$ to class $i$ following

$$
\hat{\imath}=\underset{i=1 . \ldots N}{\arg \min } d\left(C_{x}^{\phi}, \bar{C}_{i}^{\phi}\right)
$$

where

$$
\bar{C}_{i}^{\phi}(t, f)=\frac{1}{n_{i}} \sum_{k=1}^{n i} C_{x_{k}^{i}}^{\phi}(t, f)
$$

The bar in the above equation indicates the average of the TFDs over the training (learning) set. A group of $n_{i}$ signal $x_{k}^{i}$ of each class is placed in a learning set, creating the Time Frequency representation of reference for this class, $\phi$ is the kernel chosen for the Time Frequency representation. The classification method consists in measuring the distance between the Time Frequency representation of the signal and the Time Frequency representations of reference.

There are several possible various measures which can be used in the underlying problem. Each measure may yield a different classification result, as demonstrated later in Section IV. Below are the four leading distance measures which have been employed. The Euclidean distance is given by,

$$
d 2\left(C_{x}^{\phi}, \bar{C}_{i}^{\phi}\right)=\left[\iint\left|C_{x 1}^{\phi}(t, f)-\bar{C}_{i}^{\phi}(t, f)\right|^{2} d t d f\right]^{1 / 2}
$$

whereas the correlation distance is defined as

$$
\operatorname{dcor}\left(C_{x}^{\phi}, \bar{C}_{i}^{\phi}\right)=1-\frac{2 \iint C_{x}^{\phi}(t, f) \bar{C}_{i}^{\phi}(t, f) d t d f}{\left\|C_{x}^{\phi}\right\|^{2}+\left\|\bar{C}_{i}^{\phi}\right\|^{2}}
$$

On the other hand, the Kolmogorov distance is given by

$$
d \operatorname{Kol}\left(C_{x}^{\phi}, \bar{C}_{i}^{\phi}\right)=\iint\left|N C_{x}^{\phi}(t, f)-N \bar{C}_{i}^{\phi}(t, f)\right| d t d f
$$

whereas, the Bhattacharyya distance is expressed as

$$
d B h a\left(C_{x}^{\phi}, \bar{C}_{i}^{\phi}\right)=-\log \iint \sqrt{N C_{x}^{\phi}(t, f) N \bar{C}_{i}^{\phi}(t, f)} d t d f
$$

In the last two distance measures, we used the normalized TFD,

$$
N C_{x}^{\phi}(t, f)=\frac{\left|C_{x}^{\phi}(t, f)\right|}{\iint\left|C_{x}^{\phi}(s, v)\right| d s d v}
$$

Irrespective of the distance measure, the error probability of misclassification can be written as

$$
P e(d, \phi)=P[\hat{\imath} \neq i]
$$

Each class is supposed to be equally likely. We define the error as the difference of distance between the correct class $i$ and class $j$,

$$
e_{i j}=d\left(C_{x^{i}}^{\phi}, \overline{C_{j}^{\phi}}\right)-d\left(C_{x^{i}}^{\phi}, \overline{C_{i}^{\phi}}\right)
$$

It can be readily shown that,

$$
P e(d, \phi)=\frac{1}{N(N-1)} \sum_{i=1}^{N} \sum_{\substack{j=1 \\ j \neq i}}^{N} P\left[e_{i j}<0\right]
$$

Following the same reasoning as in [13], we model the error distribution as Gaussian, i.e.,

$$
P\left[e_{i j}<0\right]=\int_{-\infty}^{0} \frac{1}{\sqrt{2 \pi} \sigma_{i j}} \exp \left(-\frac{1}{2}\left[\frac{u-m_{i j}}{\sigma_{i j}}\right]^{2}\right) d u
$$

where the mean and the variance can be estimated as

$$
\begin{aligned}
& \hat{m}_{i j}=\frac{1}{n_{i}} \sum_{k=1}^{n_{i}}\left[d\left(C_{x_{k}^{\prime}}^{\phi}, \bar{C}_{j}^{\phi}\right)-d\left(C_{x_{k}^{\prime}}^{\phi}, \bar{C}_{i}^{\phi}\right)\right] \\
& \hat{\sigma}_{i j}^{2}=\frac{1}{n_{i}} \sum_{k=1}^{n_{i}}\left[d\left(C_{x_{k}^{\prime}}^{\phi}, \bar{C}_{j}^{\phi}\right)-d\left(C_{x_{k}^{\prime}}^{\phi}, \bar{C}_{i}^{\phi}\right)-\hat{m}_{i j}\right]^{2}
\end{aligned}
$$

This equation allows an estimation of the classification error which depends on the TFD and the distance measure. One may devise the best time-frequency distribution kernel that leads to a minimum classification error, but this work is beyond the scope of this paper. 


\section{EXPERIMENTAL RESULTS}

Three human gait classes were used for experimentation, 1) one-arm swing, 2) two-arms swing and 3) no-arms swing. The Doppler information for each class was captured by computing the spectrogram of the radar backscatter which is shown in Fig. 1. The data was collected at two incident angles to the walker as shown in Fig. 2. In Fig.1, the horizontal axis is time and the vertical axis represents the Doppler frequency. Positive and negative Doppler frequencies correspond to the subject moving toward or away from the radar, respectively [14]. The spine of each plot corresponds to the torso motion. Even for steady motion, the torso speeds up and slows down during a gait cycle as body goes through gait phases. This phenomenon is reflected in the slowly varying, but strong, Doppler. Arms, legs and feet motions are manifested in periodic features in the plots. For example, in Fig. 1(a), the strong peaks correspond to arm motions and the smaller peaks correspond to feet and leg motion. Composite Doppler for the legs, the arms and the feet are captured in Fig. 1(b). Note that the arm motion results in positive and negative Doppler during a gait cycle, whereas the leg motion registers positive Doppler only for the approaching moving subject and negative Doppler for receding subject. The reason is that for the approaching subjects, the legs are always moving forward, or are at most stationary, during the double limb support phase [14]. The length of the Time-Frequency window has been chosen to maximize the Renyi information [16].

\section{Preprocessing}

Before proceeding with the classification, preprocessing is performed on the raw data. First, we consider only the constant speed phase movement. Classification is not applied to acceleration and deceleration motion. So we consider, for each movement, Time-Frequency representation of 2.8 seconds which is sufficient for detecting the periodicity of the motion. Second, a time synchronization is realized. In order to take into account the different periodicity of the three different movement, we temporally synchronize the Time-Frequency representations, which is temporally translated to have arm movement beginning at the same instant. A Time-Frequency representation of reference is chosen, $C_{x}^{\varphi}(t, f)$, and time correlation of each Time-Frequency representation with the reference is computed. The maximum of the correlation gives the time translation needed for movement synchronization.

\section{Experiments, 0 degree angle}

Experiments have been conducted on five different people walking with three different motions. We first consider here the data collected at zero degree angle. Several experiments have been realized for each subject. These persons are denoted A, B, C, D, E. After preprocessing step, 5 signals are available for each person. Two scenarios are considered in the study for the formation of learning and training set.

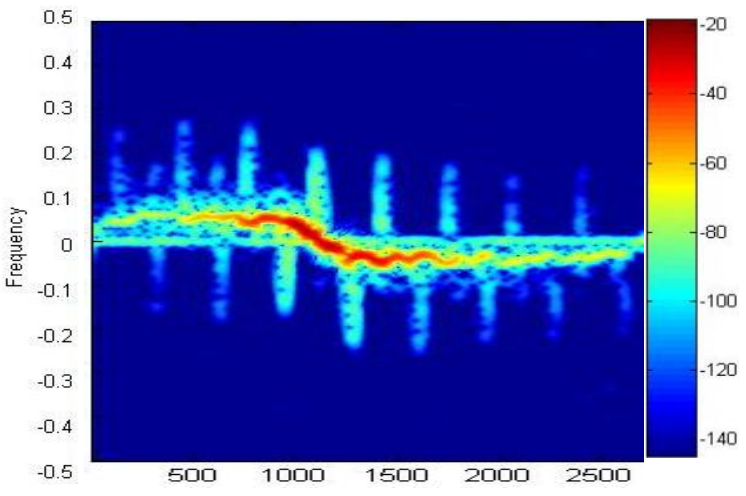

(a)

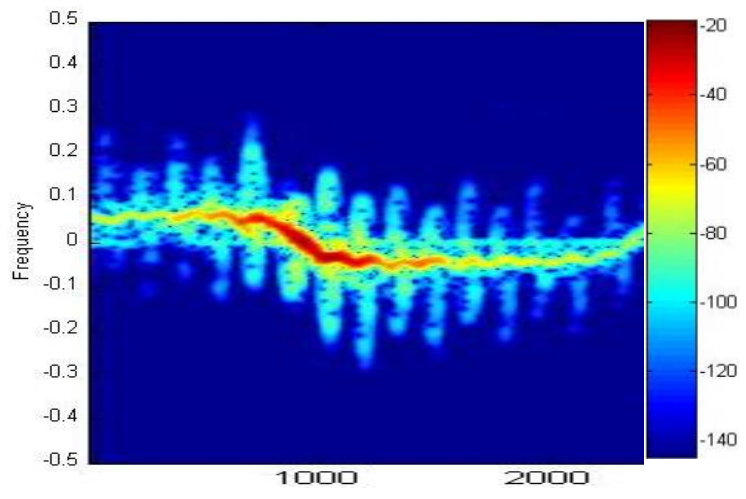

(b)

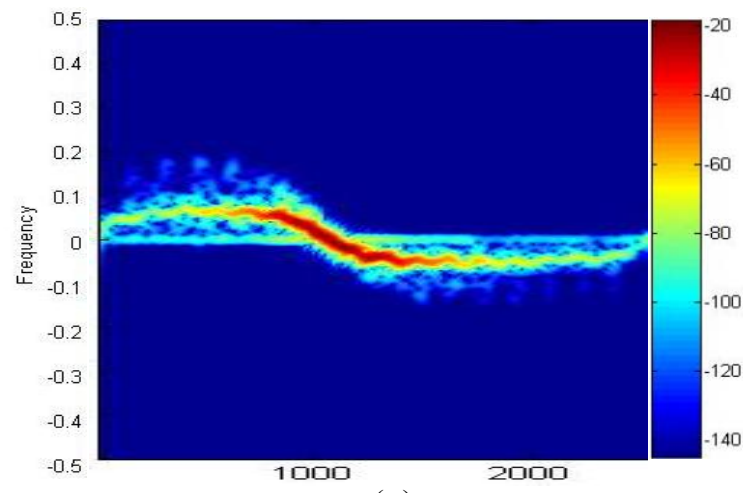

(C)

Figure 1. Spectogram for three human motions, (a) one arm swing, (b) two-arms swings, (c) no-arms swings 


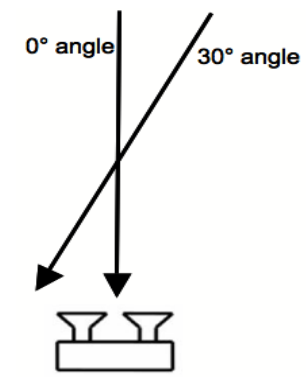

Figure 2. Data collected with two angles of incidence relative to the walker

In the first scenario, subjects $\mathrm{A}$ and $\mathrm{B}$ are used for training, whereas subjects C, D, E are used for testing. This is a realistic scenario because the three persons are unknown for the classifier. Results of the first scenario are shown in Table 1 .

\section{FiRST SCENARIO}

\begin{tabular}{|c|c|c|c|c|}
\hline 3 Classes & Error Total & Error class 1 & Error class2 & Error class3 \\
\hline $\begin{array}{c}\text { Euclidean } \\
\text { distance }\end{array}$ & $9.93 \%$ & $18.4 \%$ & $9.1 \%$ & $2.3 \%$ \\
\hline $\begin{array}{c}\text { Correlation } \\
\text { distance }\end{array}$ & $12.1 \%$ & $22.1 \%$ & $12.3 \%$ & $1.7 \%$ \\
\hline $\begin{array}{c}\text { Kolmogorov } \\
\text { distance }\end{array}$ & $10.5 \%$ & $15.5 \%$ & $9.8 \%$ & $6.1 \%$ \\
\hline $\begin{array}{c}\text { Bhattacharyya } \\
\text { distance }\end{array}$ & $13.3 \%$ & $19.6 \%$ & $8.8 \%$ & $11.6 \%$ \\
\hline
\end{tabular}

For each class and distance, we consider the total error of classification and misclassification. Class one corresponds to the one arm swinging movement; class two is for the two arms swinging movement; class three for the no arms swinging movement.

In the second scenario, for each subject, two signals of each motions are placed into the learning set. The others signals are used for the training set. In this situation, each person is known by the classifier, and we expect to have better results. Results of the second scenario are shown in Table 2 .

\section{SECOND SCENARIO}

\begin{tabular}{|c|c|c|c|c|}
\hline 3 Classes & Error Total & Error class 1 & Error class 2 & Error class 3 \\
\hline $\begin{array}{c}\text { Euclidean } \\
\text { distance }\end{array}$ & $6.94 \%$ & $1.3 \%$ & $14.4 \%$ & $5.0 \%$ \\
\hline $\begin{array}{c}\text { Correlation } \\
\text { distance }\end{array}$ & $8.41 \%$ & $2.0 \%$ & $19.1 \%$ & $4.1 \%$ \\
\hline $\begin{array}{c}\text { Kolmogorov } \\
\text { distance }\end{array}$ & $7.32 \%$ & $0.9 \%$ & $11.6 \%$ & $9.4 \%$ \\
\hline $\begin{array}{c}\text { Bhattacharyya } \\
\text { distance }\end{array}$ & $8.87 \%$ & $0.5 \%$ & $13.5 \%$ & $12.6 \%$ \\
\hline
\end{tabular}

In all four measures, and for both scenarios, the no-arm swing class assumes the lowest error. It is also evident from Table 1 that the Euclidean distance gives, in most cases, the best performance.

\section{Experiments, 30 degrees angle}

In the second example, we conducted the same experiments; but for the five people walking with an angle of 30 degrees towards the radar line of sight. The learning set is composed of the signal acquired at 0 degree angle. The classification testing signals acquired at 30 degrees angle. Figure 3 shows the TFD for the three movements we consider. For class 1, one arm swing movement, the movement of the limb is difficult to observe in the TFD because the limb is hidden by the body during the motion. Table 3 depicts the results.

\section{DEGREES ANGLE}

\begin{tabular}{|c|c|c|c|c|}
\hline 3 Classes & Error Total & Error class 1 & Error class2 & Error class3 \\
\hline $\begin{array}{c}\text { Euclidean } \\
\text { distance }\end{array}$ & $50.9 \%$ & $64.7 \%$ & $88.2 \%$ & $0.00 \%$ \\
\hline $\begin{array}{c}\text { Correlation } \\
\text { distance }\end{array}$ & $55.8 \%$ & $76.5 \%$ & $92.1 \%$ & $0.00 \%$ \\
\hline $\begin{array}{c}\text { Kolmogorov } \\
\text { distance }\end{array}$ & $52.9 \%$ & $64.7 \%$ & $94.1 \%$ & $0.00 \%$ \\
\hline $\begin{array}{c}\text { Bhattacharyya } \\
\text { distance }\end{array}$ & $45.1 \%$ & $41.2 \%$ & $82.3 \%$ & $11.7 \%$ \\
\hline
\end{tabular}

It is clear that class 3 motion does not suffer form the aspect angle, and it assumes a very low probability of error. Other classes, however, show significant errors. It appears that misclassified signals of class 1 migrate to class 3 . The movement of the arm is not recognized.

\section{CONCLUSION}

In this paper, a time-frequency classifier has been introduced for human gait classifications. Three specific motions have been studied, corresponding to three different scenarios of arm motions which describe free and confined arm swings. The microDoppler signature in the timefrequency domain of each motion style is used as a feature and is incorporated in classification distance measures between the test data $\mathrm{t}-\mathrm{f}$ distribution and the training average $\mathrm{t}-\mathrm{f}$ distributions. Two scenarios of training/testing are considered. One used all objects (people) for training, whereas the other used only a subset of objects of training and a different subset for testing. It is shown that in both cases, the time-frequency classifier gives desirable results, yielding low probability of classification errors.

\section{ACKNOWLEDGMENT}

This work by M. Amin was supported by ONR, grant no. N00014-10-1-0455. The work by C. Ioana was performed at Villanova University under ONR GLOBAL VSP, grant no. N62909-09-1-4082. 


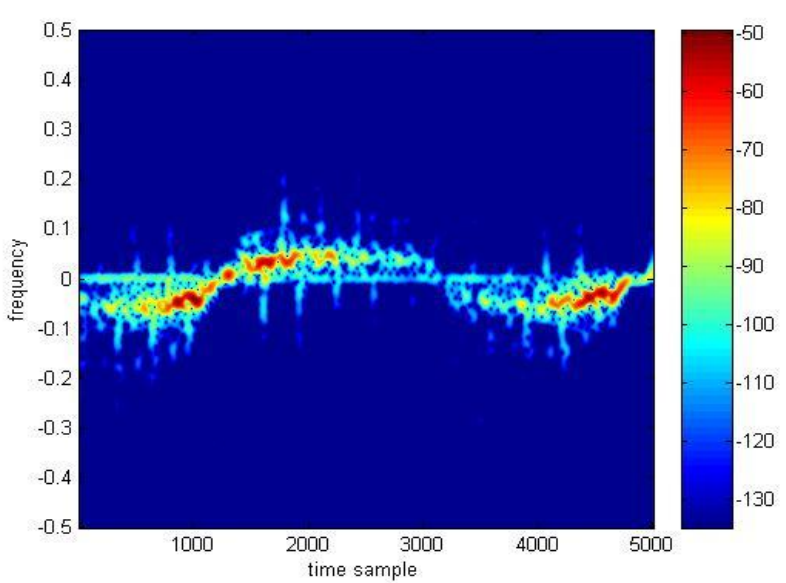

(a)

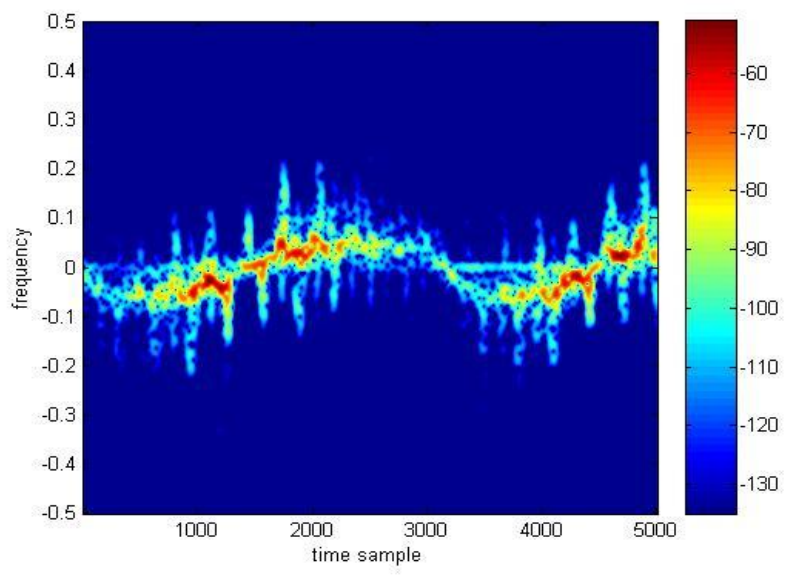

(b)

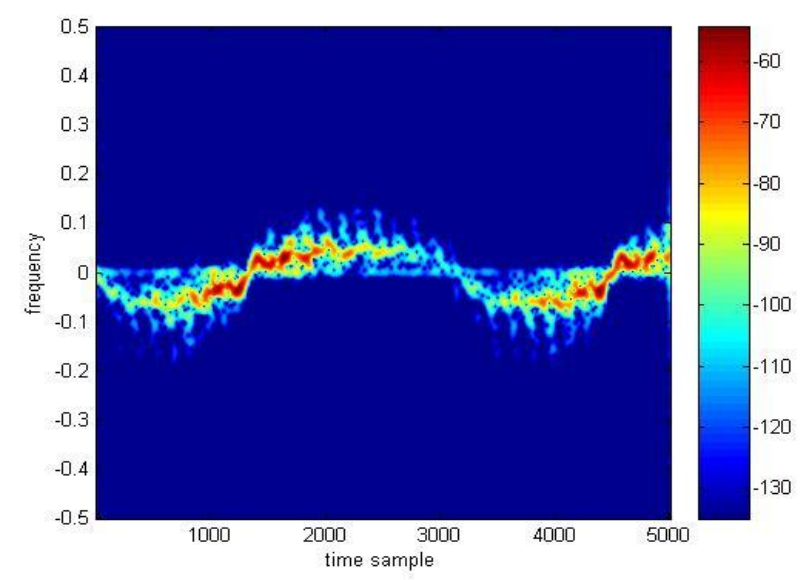

(c)

Figure 3. Spectogram for three human motions with aspect angle of 30 degrees, (a) one arm swing, (b) two-arms swings, (c) no-arms swings

\section{REFERENCES}

[1] V. Chen, F. Li, S.-S. Ho, and H. Wechsler, "Analysis of micro-Doppler signatures," Radar, Sonar and Navigation, IEE Proceedings - 150, pp. 271, Aug. 2003.

[2] V. Chen, "Detection and analysis of human motion by radar," Radar Conference, 2008. RADAR '08. IEEE, pp. 1 to 4, May 2008.

[3] J. Gray, "The Doppler spectrum for accelerating objects," Radar Conference, 1990., Record of the IEEE 1990 International, pp. 385 to 390, May 1990.

[4] P. Setlur, F. Ahmad, and M. Amin, "Analysis of micro-Doppler signals using linear FM basis decomposition," Radar Sensor Technology X 6210(1), p. 62100M, SPIE, 2006.

[5] P. Setlur, M. Amin, and F. Ahmad, "Urban target classifcations using time-frequency micro-Doppler signatures," Signal Processing and Its Applications, 2007. ISSPA 2007. 9th International Symposium on , pp. $1\{4, F e b .2007$.

[6] V. C. Chen, "Joint time-frequency analysis for radar signal and imaging," IEEE International Geoscience and Remote Sensing Symposium, 2007. IGARSS 2007 , pp. $5166\{5169$, July 2007.

[7] T. Thayaparan and L. Stankovic and I. Djurovic, "Micro-doppler-based target detection and feature extraction in indoor and outdoor environments," Journal of the Franklin Institute 345(6), pp. $700\{722$, 2008.

[8] S.S. Ram and H. Ling, "Analysis of microDopplers from human gait using reassigned joint time-frequency transform," Electronic Letters 43, May 2007.

[9] S. S. Ram, Y. Li, A. Lin, H. Ling, "Doppler-based detection and tracking of humans in indoor environments," Journal of The Franklin Institute 345(6), pp. $679\{699,2008$.

[10] J. L. Geisheimer, W. S. Marshall and E. Greneker, \A continuous-wave (CW) radar for gait analysis," Thirty-Fifth Asilomar Conference on Signals, Systems and Computers , pp. 834\{838, 2001.

[11] Z. Zhang, P. Pouliquen, A. Waxman and A. Andreou, "Acoustic microDoppler radar for human gait imaging," J Acoust Soc Am 121, pp. $110\{113$, March 2007.

[12] M. Davy, C. Doncarli, and F. Boudreaux-Bartels, "Improved Optimization of Time-Frequency-Based Signal Classifiers," IEEE Signal Processing Letters, February 2001.

[13] M. Otero, "Application of a continuous wave radar for human gait recognition," in Society of Photo-Optical Instrumentation Engineers (SPIE) Conference Series, 5809, pp. 538 \{548, 2005.

[14] B. Mobasseria and M. Amin, "A Time-Frequency Classifier for Human Gait Recognition," SPIE Defense, Security and Sensing, Orlando, Florida. April 13-17, 2009,

[15] Y. Kim and H. Ling, "Human activity classification based on microdoppler signatures using a support vector machine," IEEE Transactions on GeoScience and Remote Sensing, vol. 47, no. 5, pp. $1328-1337,2009$.

[16] W. Williams, M. Brown, and A. Heros III. "Uncertainty, Information, time-frequency distributions". SPIE Advanced Signal Processing Algorithms, Architectures and Implementations II, 1991 